\title{
LA PROTECCIÓN DE LA ESFERA INDIVIDUAL DEL DERECHO DE LIBERTAD RELIGIOSA: DESAFÍOS ACTUALES EN EL DERECHO CHILENO Y COMPARADO ${ }^{1}$ \\ PROTECTION OF THE INDIVIDUAL RIGHT OF RELIGIOUS FREEDOM: CURRENT CHALLENGES IN CHILE AND ABROAD
}

Miguel Sánchez-Lasheras ${ }^{2}$

Resumen: Con frecuencia se recalca la importancia de proteger el derecho de libertad religiosa, pero, sin embargo, se desconoce el contenido específico de este importante derecho fundamental. En el presente artículo se exponen, de manera sintética, los derechos y libertades que conforman la dimensión individual de la libertad religiosa. Los principales ejemplos de normas positivas se refieren a la República de Chile, si bien se traen a colación algunos temas de actualidad en el contexto internacional y comparado,

Palabras clave: Libertad religiosa. Derechos individuales. Derecho chileno. Derecho comparado.
Abstract: The importance of protecting the right to religious freedom is often stressed, but nonetheless, the content of this important fundamental right is sometimes unknown. This article tries to expose, in a synthetic way, the rights and freedoms that shape the individual dimension of religious freedom. The main examples of positive laws refer to the Republic of Chile, although some current issues in the international and comparative context are also mentioned.

Keywords: Religious freedom. Individual rights. Chilean law. Comparative law.

\footnotetext{
1 El presente trabajo se basa, en parte, en mi ponencia "La dimensión individual del derecho de libertad religiosa: desafíos actuales", dictada en el Seminario internacional "Los temas emergentes en derechos fundamentales", organizado por el Departamento de Derecho Público de la Universidad de Talca (17 y 18 de agosto de 2017).

Doctor Europeo en Derecho por la Universidad de Navarra (España); Profesor asociado de Historia del Derecho y de Derecho Canónico de la Pontifícia Universidad Católica de Valparaíso; Profesor de la Escuela de Derecho de la Pontificia Universidad Católica de Valparaíso (Chile). Avenida Brasil, 2950, segundo piso, Valparaíso; miguel.sanchez@pucv.cl
} 


\section{Introducción}

En la tradición jurídica occidental, especialmente la norteamericana, la libertad religiosa se ha concebido históricamente como "la primera de las libertades" (Rubio López, 2006). En ella se contienen dos dimensiones referidas a esferas de protección diferentes: el principio y el derecho.

Así, la libertad religiosa como principio se refiere al modo en que el Estado se relaciona con lo religioso: se declara su radical incompetencia para definirse en esta materia, al entenderse que dicha definición le corresponde en exclusiva a los ciudadanos (Martín de Agar, 2003, pp. 338-339).

Por otro lado, toda persona es titular del derecho de libertad religiosa por exigencia misma de su propia naturaleza (Martín de Agar, 2016, p. 196). El contenido de este derecho es extenso e incluye la protección de otros que están directa o indirectamente vinculados a él, tal y como ha ido concretándose en los principales textos internacionales de protección de los derechos humanos. Instrumentos como la Declaración Universal de 1948, el Convenio Europeo de 1950 o la Convención Americana (Pacto de San José) de 1969, aluden al concepto de religión en un sentido amplio, incluyéndose a las religiones tradicionales, a los nuevos movimientos religiosos y a las creencias con perfiles similares a las de las religiones tradicionales. ${ }^{3}$

La doctrina científica, por su parte, ha conceptualizado nítidamente la tríada de libertades recogidas en el artículo 18 de la Declaración Universal de Derechos Humanos, a saber, libertad de pensamiento, libertad de conciencia y libertad de religión. ${ }^{4}$ Aunque cada una de ellas tiene su objeto propio, las tres comparten un mismo fundamento: la libertad inherente a la dignidad de la persona, siendo la potencia intelectual la que le diferencia significativamente del resto de seres vivos. ${ }^{5}$

De este modo, se entiende por libertad ideológica la facultad de manifestar, defender y propagar las propias ideas; la libertad de conciencia supone la garantía de poder actuar y obrar sin ser perturbado por los poderes públicos; $y$, la libertad religiosa puede definirse como el derecho de toda persona a poder vivir en conformidad o en disconformidad con sus convicciones o creencias religiosas (Chapman, 2013; González Merlano, 2015, p. 84-88; Olmos Ortega, 2009, p. 6).

Volviendo al concepto de religión en un sentido amplio, conviene identificar su contenido y los elementos específicos que lo definen, que son básicamente tres: ${ }^{6}$ la fe religiosa o creencia, el sentido de pertenencia o identidad y el estilo de vida:

\footnotetext{
3 Obviamente, las denominadas "sectas destructivas" quedan excluidas de esta protección, en la medida en que atentan contra la dignidad del ser humano o contra el orden público. Además, la legislación de algunos países, como España y Perú, prohíbe expresamente la asimilación de los fines religiosos con otros fines que pudieran estar más o menos relacionados (vid. el artículo 5 párrafo segundo de la Ley N²9.635 de la República del Perú).

4 Declaración Universal de Derechos Humanos (1948), artículo 18.

5 Lo cual no obsta para que deba respetarse a los animales y al resto de seres vivos; pero, obviamente, dicho respeto no puede ser equiparado con la dignidad humana.

6 Seguiremos el esquema propuesto por Olmos Ortega (2016, p. 7).
} 
a) Fe religiosa o creencia: incluye a su vez a la no creencia. Las creencias pueden integrar convicciones o valores acerca de lo divino o del destino espiritual de la humanidad. Se incluyen las teístas, no teístas y ateas, así como el derecho a no profesar ninguna religión ni creencia.

b) Sentido de pertenencia o identidad: puede estar compuesto por creencias, culto, ritos y tradiciones. Dicho de otra manera, la identidad implica formar parte de una comunidad que se identifica con unas creencias determinadas.

c) Estilo de vida: se compone de las tradiciones y prácticas religiosas relacionadas con las festividades, la vestimenta, la alimentación o determinados rituales vinculados con diferentes hitos vitales, entre otros ejemplos.

Cabe advertir que el sujeto titular del derecho de libertad religiosa no es solamente la persona - el individuo -; también los grupos - es decir, las confesiones religiosas - son titulares de este derecho fundamental y pueden exigir a los poderes públicos que garanticen su ejercicio en caso de que este se vea impedido o menoscabado. Sin embargo, en el presente trabajo nos centraremos en analizar la libertad religiosa en su dimensión individual.

\section{El contenido de la dimensión individual del derecho de libertad religiosa}

Como se ha apuntado, toda persona, por el mero hecho de serlo, es titular del derecho de libertad religiosa, si bien el reconocimiento de los derechos y libertades individuales que se vinculan a este derecho fundamental varía según el texto normativo que se tome como modelo. ${ }^{7}$ En este caso se ha optado por tomar como guía el artículo 2 de la Ley Orgánica de Libertad Religiosa española, ${ }^{8}$ que recoge una interesante distinción entre cuatro libertades que, a su vez, implican la protección de otras libertades y prerrogativas.

Así, aludiremos al contenido fundamental de la libertad religiosa personal; la libertad de culto y asistencia religiosa; la libertad de información y enseñanza religiosa; y, la libertad de reunión, manifestación y asociación.

\subsection{Libertad religiosa personal}

Se trata de las actitudes que el individuo, aisladamente considerado, puede adoptar ante el fenómeno religioso. Consiste tanto en actitudes positivas -se reconoce el derecho de toda persona a

\footnotetext{
De acuerdo con Martín de Agar (2016, p. 190), la libertad religiosa es considerada en gran parte de los documentos sobre derechos fundamentales como derecho individual; lo que resulta lógico, habida cuenta de que la religión atañe ante todo a las actitudes, sentimientos y decisiones más íntimas de la persona.

8 Ley Orgánica del Reino de España 1/1980, de 5 de octubre. Su contenido está prolijamente detallado en Ciáurriz (1994, pp. 429-468).
} 
profesar las creencias religiosas que libremente elija- como en actitudes negativas -no profesar religión alguna, cambiar de una confesión a otra o abandonar su credo religioso ${ }^{9}$ - Se protege, además, tanto su difusión como su no difusión; es decir, se ampara la libertad para manifestar libremente las propias creencias y para abstenerse de declarar sobre ellas.

\subsection{Libertad de culto y asistencia religiosa}

El culto y la asistencia suelen confundirse porque habitualmente aparecen unidos, pero es claro que ambas actividades tienen objetos diferentes. Aquí nos referiremos a la celebración de festividades, la celebración de ritos matrimoniales y la recepción de sepultura conforme a las prescripciones de cada confesión.

\section{a) Conmemoración de festividades}

Consiste en la facultad de celebrar los actos de culto y las manifestaciones de índole personal o colectiva que cada confesión religiosa estime como propias. Los límites a esta facultad son únicamente el orden público y los propios de los derechos de reunión y manifestación.

Puede ocurrir que las confesiones religiosas aspiren a que algunas de sus festividades sean reconocidas por el Estado y se conviertan en día feriado con carácter oficial. No en vano, en los países occidentales de tradición cristiana se reconoce el domingo como día de descanso semanal. En Chile, hay varias festividades católicas que son feriado en todo el país, como la Virgen del Carmen (16 de julio), la Asunción de la Virgen (15 de agosto) o la Inmaculada Concepción (8 de diciembre), entre otras. A su vez, desde el año 2008 se reconoce el día 31 de octubre como día feriado con motivo del "Día de las Iglesias evangélicas". ${ }^{10}$

\section{b) Celebración de los ritos matrimoniales}

No cabe duda que el matrimonio y la familia revisten una especial significación para las diferentes tradiciones religiosas. En casi todas ellas se atribuye un carácter sagrado a la relación matrimonial entre un hombre y una mujer, que es la base sobre la que se construye la familia. Esta, a su vez, se entiende y protege como la "célula básica de la sociedad". ${ }^{11}$ De hecho, la defensa de la familia y, en particular, del matrimonio entre un hombre y una mujer se ha convertido, en los últimos tiempos, en uno de los asuntos que mayor consenso ha generado entre las autoridades de diversos credos religiosos. ${ }^{12}$

\footnotetext{
9 En este punto, es necesario recordar que el abandono formal de la propia religión o apostasía sigue estando penado con la cárcel, e incluso con la pena de muerte, en no pocos países africanos y asiáticos (vid. Gas Aixendri, 2012).

10 Ley N²0.299 de la República de Chile, establece feriado el 31 de octubre, Día Nacional de las Iglesias Evangélicas y Protestantes.

11 La propia Constitución chilena de 1980 , en su artículo $1^{\circ}$, se refiere a la familia como el núcleo fundamental de la sociedad.

12 La doctrina de la Iglesia católica sobre esta materia ha sido firme y bien conocida a lo largo de su historia. En la actualidad, es preciso tener en cuenta la creciente influencia del protestantismo en América Latina (vid. Parker, 2013). Sobre la postura
} 
Por otro lado, la mayoría de las confesiones religiosas tiene sus propias normas y preceptos referidos al estado objetivo instaurado por el vínculo matrimonial que une de manera estable al hombre y a la mujer. En el caso de la Iglesia católica, es bien conocida la relevancia que ha tenido el Derecho canónico en esta materia, y no únicamente ad intra sino también ad extra de la propia comunidad eclesial. ${ }^{13}$

c) Derecho a recibir sepultura digna sin discriminación por motivos religiosos

En la actualidad, referirse a asuntos como la regulación de las sepulturas o de los cementerios supone aludir a un tema prácticamente tabú: la realidad de la muerte. No parece, por tanto, que su regulación constituya un asunto de especial importancia, ni mucho menos que sea debatida en la esfera pública como un tema de interés nacional. Sin embargo, esto no ha sido siempre así; al menos en Chile, donde las denominadas "leyes laicas" de finales del siglo XIX-cuya normativa implicaba, entre otros asuntos, la secularización de los cementerios- fueron objeto de apasionadas discusiones entre las autoridades eclesiásticas y los dirigentes políticos de la época (Salinas, 2016, pp. 52-54). ${ }^{14}$

Lo cierto es que las confesiones religiosas siguen otorgando un valor fundamental a la realidad mortal del ser humano y a la promesa de una vida futura. Es más, una de las principales razones de ser de los diferentes credos, si no la fundamental, es precisamente la esperanza de trascender esta vida para alcanzar otra mejor. Todo ello tiene como consecuencia que, por lo general, las personas que profesan una determinada fe religiosa deseen ser enterradas de acuerdo con los ritos y normas propias de su propia confesión. ${ }^{15}$ El caso de los fieles musulmanes es, en este sentido, paradigmático. ${ }^{16}$

d) No ser obligado a practicar actos de culto o a recibir asistencia religiosa contraria a las propias convicciones personales

Se garantiza la libertad religiosa de aquellas personas sometidas al régimen de algún establecimiento o institución pública, como fuerzas armadas, hospitales, cárceles y centros docentes, entre otros. Incluye también la libertad del individuo que, aun compartiendo una determinada cre-

\footnotetext{
de los evangélicos chilenos en torno a los denominados "temas valóricos", entre los cuales se encuentra el matrimonio y la familia, puede verse el interesante estudio sociológico de Fediakova e Parker (2009, pp. 65-71).

13 En materia de familia, la influencia que el Derecho canónico ha tenido en las legislaciones civiles es notoria. Basta comprobar cómo, en asuntos como los impedimentos matrimoniales o la manifestación del consentimiento, algunas legislaciones civiles son prácticamente una copia de la normativa canónica (vid. Salinas, 2006, pp. 27-36).

14 Vid.

15 No obstante, y por lo general, la legislación en esta materia adolece de explicitud y no prevé explícitamente que las confesiones religiosas puedan habilitar sus propios cementerios. Así ocurre, por ejemplo, con la ley española de libertad religiosa. Para remediar dicha laguna legal, esta materia suele ser objeto de desarrollo específico en los acuerdos de cooperación que los Estados pueden suscribir con las confesiones religiosas, al tratarse de un asunto muy específico, pero no por ello menos importante para los fieles, independientemente de la tradición religiosa a la que estén adscritos.

16 A modo de ejemplo, puede consultarse el artículo 2, epígrafe quinto, del Acuerdo de Cooperación del Estado Español con la Comisión Islámica de España, de 1992.
} 
encia religiosa, opta por no practicar el culto que eventualmente se ofrezca en el contexto de dichas instituciones. ${ }^{17}$

\subsection{Información y enseñanza religiosa}

En este punto, es importante diferenciar dos aspectos fundamentales: el derecho a recibir o a impartir información religiosa de toda índole, por un lado; y, por otro, el derecho de los padres a elegir para sus hijos la educación religiosa y moral que esté de acuerdo con las propias convicciones.

a) Derecho a recibir o a impartir información religiosa de toda indole

A priori, la normativa que regula el papel de los medios de comunicación social debe posibilitar a los individuos y a los grupos el acceso a dichos medios, sin que en ningún caso pueda darse discriminación entre los diferentes colectivos, incluidos los de naturaleza o configuración religiosa. Este derecho lleva consigo la facultad de réplica en medios ajenos, así como el derecho de acceso a la prensa, radio y televisión públicas. ${ }^{18}$

b) Derecho a la elección de la educación religiosa y moral que esté de acuerdo con las propias convicciones

La Historia demuestra que la posibilidad de elegir con libertad un determinado tipo de enseñanza, acorde con las creencias y convicciones de los padres, no es un asunto pacífico, ya que existen diferentes visiones en torno al papel que le corresponde desempeñar al Estado y a los grupos intermedios en la provisión de servicios educativos (Celis \& Zárate, 2015, pp. 3-5).

En lo que respecta a los centros educativos no estatales, es bien conocido el papel desempeñado por la Iglesia católica y por las diferentes Iglesias evangélicas en el campo de la enseñanza, no solamente en los países de tradición cristiana sino también en los denominados "países de misión”. Sin ir más lejos, el 44 \% de los alumnos chilenos en edad escolar acuden a un centro educativo religioso. ${ }^{19}$

\subsection{Derechos de reunión, manifestación y asociación}

Se trata de derechos que, si bien se ejercitan por la persona individualmente considerada, responden a su carácter de "ser social por naturaleza". Su reconocimiento es necesario para que esta

\footnotetext{
17 En el caso chileno, son varios los reglamentos que prevén la cooperación entre el Estado y las confesiones en materia de asistencia religiosa (vid. Sánchez-Lasheras, 2016, pp. 173-174).

18 A modo de ejemplo, cada domingo la televisión pública española incluye programación religiosa vinculada a diferentes credos, retransmitiendo la misa católica o difundiendo reportajes sobre las diferentes actividades promovidas por grupos evangélicos, judíos y musulmanes (vid. < http://www.rtve.es/alacarta/programas/tve/religiosos/1/>).

19 Y, dentro de ese porcentaje, la mayor parte lo hacen en instituciones de la Iglesia católica romana (vid. Celis \& Zárate, 2015, pp. 5-7).
} 
dimensión surta efectos en el ámbito civil, sin que pueda ser restringida salvo en los casos en que colisione con otros derechos fundamentales o en que se actúe contra el orden público o la ley.

Desde el punto de vista práctico, la regulación de la vertiente específicamente religiosa de estos derechos es reconducida, nuevamente, a la legislación estatal común. No obstante, cabe la posibilidad de que se reconozca de forma específica el asociacionismo religioso, es decir, la existencia de asociaciones promovidas por confesiones religiosas.

De hecho, en Chile, tal y como se apunta en un informe recientemente publicado por el profesor Del Picó (2017) sobre la evaluación del sistema jurídico de reconocimiento del fenómeno religioso, "la opción de crear personas jurídicas derivadas de la entidad matriz es significativamente conocida y utilizada por parte de las confesiones, lo que se refleja en que la quinta parte de las entidades religiosas han hecho uso de la normativa pertinente." (p. 52). ${ }^{20}$

\section{Desafíos actuales de la libertad religiosa en Chile}

Si analizamos la realidad chilena actual, la primera afirmación que puede hacerse es que en líneas generales existe un amplio reconocimiento jurídico y una protección suficiente de la libertad religiosa. Así lo perciben las propias entidades implicadas: un 80 \% de ellas declara no haber sido afectadas en el ejercicio de este derecho fundamental (DEL PICÓ RUBIO, 2017, pp. 22-26).

Entre las entidades que declaran haber sido afectadas - el $20 \%$ restante - una parte significativa asegura haber visto obstaculizado su derecho a recibir e impartir enseñanza o información religiosa, así como el derecho a fundar templos y lugares con significación religiosa. También se han sentido vulnerados el derecho a la asistencia religiosa y a la libre manifestación de las creencias (Del Picó Rubio, 2017, pp. 24-25).

Con todo, en la actual discusión jurídico-política existen algunos asuntos que aparecen directa o indirectamente vinculados con la libertad religiosa. Entre otros, cabe apuntar los siguientes:

\section{a) Posible actualización de la denominada "Ley de cultos"}

La Ley 19.638, de 1999, relativa a la constitución jurídica de iglesias y entidades religiosas y conocida como "Ley de cultos", constituye la principal regulación específica sobre esta materia. ${ }^{21}$ Su posible reforma - planteada en varias ocasiones- se centraría, sobre todo, en el funcionamiento del Registro de Entidades Religiosas (Precht, 2015). A mi juicio, el Consejo Asesor Interreligioso, impulsado recientemente por la Oficina Nacional de Asuntos Religiosos (ONAR) (U.S Department of State (2015, p. 4), podría resultar útil en este sentido si consigue aunar los intereses de las

\footnotetext{
20 Se trata de un estudio sistemático y riguroso - el primero de su naturaleza en Chile - sobre la percepción de las propias entidades religiosas acerca del régimen legal vigente. También se aportan datos objetivos sobre la labor educativa y social de las diferentes confesiones con presencia en Chile.

21 Ley de la República de Chile No 19.638, de 14 de octubre de 1999.
} 
diferentes confesiones ante una eventual actualización de la ley que más directamente les afecta en su conjunto.

b) Reconocimiento de la objeción de conciencia a raiz de la despenalización del aborto en tres causales

La reciente promulgación de la ley que despenaliza el aborto en tres causales ${ }^{22}$ ha puesto de manifiesto la importancia del reconocimiento de la objeción de conciencia, tanto individual como colectiva. Como ejemplo de lo anterior, cabe apuntar que el rector de la Pontificia Universidad Católica de Chile (PUC) señaló en varias ocasiones que las clínicas vinculadas a dicha casa de estudios no practicarían abortos, con independencia de que el proyecto de ley que se tramitaba en el Congreso reconociera o no la objeción de conciencia institucional. ${ }^{23}$ Aunque el Tribunal Constitucional resolvió en favor de abrir las puertas a este reconocimiento, ${ }^{24}$ todavía se desconoce cómo va a resultar su aplicación ante los eventuales conflictos que puedan suscitarse en la práctica.

c) Existencia de controversias judiciales y de conflictos relacionados con los pueblos originarios, especialmente el mapuche

En el contexto de los llamados pueblos originarios o indígenas, el hecho religioso aparece inseparablemente unido a la "madre tierra" y a los recursos naturales, que tienen un carácter sagrado -y, por tanto, central- en su cosmovisión (Sánchez-Lasheras, 2016, pp. 177-178). Parece claro que la sociedad chilena ha evolucionado en esta materia y, en la actualidad, los individuos y las comunidades indígenas tienen una mayor conciencia de los mecanismos legales de que disponen para hacer valer sus derechos; de ahí que los conflictos judiciales por asuntos relacionados con la cosmovisión indígena, especialmente la mapuche, hayan aumentado notablemente en los últimos años (Instituto Nacional de Derechos Humanos de Chile, 2016, pp. 10-11).

Por otro lado, se hace necesario aludir al preocupante aumento de episodios de violencia sobre templos católicos y evangélicos en la región de Araucanía. Son hechos sin precedentes en Chile y se deben, en parte, a que las tensiones en dicha región tienen un importante componente étnico-religioso (Instituto Nacional de Derechos Humanos de Chile, 2016, pp. 160-165).

\footnotetext{
${ }_{22}$ Ley de la República de Chile $N^{\circ} 21.030$, de 23 de septiembre de 2017, que regula la despenalización de la interrupción voluntaria del embarazo en tres causales.

23 Sobre la posición de la rectoría de la Pontificia Universidad Católica de Chile en torno a este asunto, vid. <http://rectoria. uc.cl/actividades-y-eventos/rector-pide-ampliar-la-objecion-de-conciencia-a-todo-el-equipo-medico > .

24 Vid. el fallo del Tribunal Constitucional de 28 de agosto de 2017, Rol Nº 3729 (3751)-17-CPT; y, en particular, el resumen sobre el tema de la objeción de conciencia disponible en: < https://www.tribunalconstitucional.cl/app/webroot/files/files/ Sintesis_2doCapitulo_Rol_3729(3751).pdf>.

Puede encontrarse un buen compendio de trabajos académicos con argumentos técnico-jurídicos en la publicación colectiva coordinada por Casas Becerra y Lawson (2016).
} 


\section{Desafíos actuales de la libertad religiosa en el mundo}

Desde una óptica comparada, no son pocos los desafíos que se presentan en relación con la protección de la libertad religiosa, si bien no todos revisten la misma gravedad. A mi juicio ${ }^{25}$ dichos desafíos pueden englobarse en tres grandes bloques:

a) Ausencia o restricciones al reconocimiento del derecho fundamental de libertad religiosa

Tal y como se apunta en el último informe elaborado por la Comisión Estadounidense para la Libertad Religiosa Internacional (USCIRF), ${ }^{26}$ existe un elevado número de Estados que merecen una atención especial por violaciones sistemáticas a la libertad religiosa; principalmente en África y Asia, aunque no faltan menciones a países europeos. ${ }^{27}$

El informe destaca que la esfera individual de la libertad religiosa de las personas debe ser salvaguardada en todo caso, independientemente de su pertenencia a un grupo religioso mayoritario, o minoritario, o su no pertenencia a ninguno (United States Comission on International Religious Freedom (2017, p. 11). La actual crisis de los rohingyas en Myanmar ${ }^{28}$ es un ejemplo de persecución de una minoría religiosa - la musulmana- en un país con una fe mayoritaria -la budista- donde se mezclan, además, otros factores de tipo étnico y político (United States Comission on International Religious Freedom (2017, pp. 22-27).

b) Instrumentalización de las creencias religiosas para justificar el uso de la violencia con fines políticos o en discursos marcadamente nacionalistas y étnicos

Los atentados perpetrados en los últimos años por individuos y grupos fundamentalistas islámicos, así como la creación del Estado Islámico (ISIS) son ejemplos de cómo una determinada fe religiosa puede ser utilizada y tergiversada con fines violentos para conseguir objetivos que trascienden los límites de la religión. La resolución de este tipo de conflictos resulta más compleja, precisamente por la multitud de variables que inciden en su surgimiento y en la manera de enfocarlos (United States Comission on International Religious Freedom, 2017, pp. 1-2, 90-95).

Asimismo, las creencias pueden ser invocadas en favor de la cohesión social o como reforzamiento de la identidad de un determinado pueblo o nación. Por ejemplo, en los países de Europa oriental la llegada de la democracia en la década de los noventa del siglo pasado trajo consigo el resur-

\footnotetext{
25 En la estructuración de dichos retos o desafíos, coincido en lo sustancial con el discurso del Papa Francisco ante la comunidad hispana de los Estados Unidos (vid. Francisco, 2015).

26 Se trata de un organismo asesor independiente del Gobierno de los EE. UU., separado del Departamento de Estado, que supervisa la libertad religiosa en el extranjero y hace recomendaciones de política al presidente, al secretario de estado y al congreso. Vid. http://www.uscirf.gov/reports-briefs/annual-report.

27 Se trata de los siguientes países: Afganistán, Arabia Saudí, Azerbayán, Baréin, China, Corea del Norte, Cuba, Egipto, Eritrea, India, Indonesia, Irán, Irak, Kazajistán, Laos, Malaysia, Myanmar, Nigeria, Pakistán, República Centroafricana, Rusia, Sudán, Siria, Tajikistán, Turquía, Turkmenistán, Uzbekistán y Vietnam (vid. United States Comission on International Religious Freedom, 2017, p. 4).

28 También conocido como Burma.
} 
gimiento del hecho religioso, que en algunos casos aparecía entrelazado con aspectos relevantes de la cultura y de la idiosincrasia locales (Pankhurst \& Kilp, 2013, p. 234). Así, no es infrecuente que el discurso nacionalista o étnico instrumentalice las creencias religiosas más para reforzarse a sí mismo que para promover de un modo genuino la libertad de religión y de culto. ${ }^{29}$

c) Reducción de la libertad religiosa a una subcultura sin derecho a voz y voto en la plaza pública

La laicidad del Estado implica la neutralidad o incompetencia de los poderes públicos en materia religiosa. En cambio, el laicismo ideológico mantiene una actitud beligerante ante la presencia pública de las religiones - principalmente las monoteístas- que son percibidas como una amenaza para la paz y la convivencia social. ${ }^{30}$ Así entendido, el laicismo es contrario al principio de neutralidad, pues supone adoptar una postura ideológica militante contra el hecho religioso. ${ }^{31}$

En esta línea, se ha argumentado que la religión no debería ser objeto de un tratamiento jurídico específico, es decir, diferenciado del que se otorga a las creencias humanistas, filosóficas o no religiosas. ${ }^{32}$ Sin embargo, podría argumentarse que la libertad religiosa comprendida en su sentido negativo, es decir, la no interferencia del Estado, sería insuficiente. La apuesta, entonces, sería apuntar hacia un concepto positivo de libertad, que suponga la gestión y la promoción del factor religioso en su conjunto por parte del Estado (González Merlano, 2015, p. 101); en definitiva, el reconocimiento de una libertad no indiferente sino cooperante (Rossell, 2015, pp. 121-125).

\section{Consideraciones finales}

La protección de la esfera individual del derecho de libertad religiosa resulta insuficiente si, al mismo tiempo, no se considera su dimensión colectiva e institucional. Por otro lado, la libertad de las personas para profesar la fe que consideren oportuna, o para no profesar ninguna creencia, es solamente una de las facultades comprendidas dentro del ámbito de protección de la dimensión individual de la libertad religiosa. Así, debe tenerse en cuenta el culto y la asistencia religiosa, que incluyen la celebración de festividades, la celebración de ritos matrimoniales y la recepción de sepultura conforme a las prescripciones de cada confesión. Cabe aludir también a la información y a la enseñanza religiosa, así como a los derechos de reunión, de manifestación y de asociación.

\footnotetext{
29 Varios países de la península balcánica y de Europa oriental pueden ser mencionados como ejemplo de confusión entre elementos de tipo político, étnico y religioso (vid. Flere, Lavric, \& Djordjevic, 2017).

30 En sentido contrario, argumenta el Papa Francisco (2015): "La libertad religiosa, sin duda, comporta el derecho de adorar a Dios, individualmente y en comunidad, de acuerdo con la propia conciencia. Pero, por otro lado, la libertad religiosa, por su naturaleza, trasciende los lugares de culto y la esfera privada de los individuos y las familias, porque el hecho religioso, la dimensión religiosa, no es una subcultura, es parte de la cultura de cualquier pueblo y de cualquier nación." (p. 2).

31 Vid. González Merlano (2015). El autor afirma que su país -Uruguay- sería un ejemplo de Estado laicista, donde a lo largo del siglo XX "se instaló una actitud de prescindencia hacia lo religioso, una especie de libertad de indiferencia. Esto sucede cuando se confunde el principio de laicidad con la ideología laica negativa - el laicismo; presentando esta, en definitiva, un desconocimiento de la realidad, pues se legisla de acuerdo a una visión parcializada, donde se excluye al fenómeno religioso del horizonte cultural." (p. 98).

32 Vid. Koppelmann (2006, pp. 572-573).
} 
En el caso de Chile, existe una protección suficiente de la libertad religiosa, tanto en su dimensión individual como desde el punto de vista institucional y colectivo. Ello no implica la ausencia total de discriminación o de incidencias relacionadas con este asunto; pero, en líneas generales, puede afirmarse que los poderes públicos son sensibles al reconocimiento y a la tutela del conjunto de manifestaciones de fe trascendente presentes en la sociedad chilena. No obstante, la celeridad de los cambios sociales y la globalización de algunas tendencias legislativas -como, por ejemplo, la despenalización del aborto en determinadas causales- nos obligan a seguir planteándonos algunas preguntas en materia de libertad religiosa individual; como, por ejemplo, en relación con el reconocimiento o no de la objeción de conciencia, y en qué condiciones.

Desde una óptica comparada, son dos los extremos que conviene evitar: por un lado, la instrumentalización de las creencias religiosas para justificar el uso de la violencia o para dar sustento a discursos políticos de tipo étnico o identitario; y, por otro, la negación de toda presencia de las religiones en el ámbito civil, relegándolas a una especie de subcultura sin derecho a voz y voto en la plaza pública. En todo caso, el reconocimiento formal y la protección material de la libertad religiosa sigue siendo un tema pendiente en la práctica de muchos países africanos y asiáticos. De hecho, y a pesar de las diferentes vicisitudes históricas, el reconocimiento de la libertad religiosa encuentra sus raíces en la tradición jurídica occidental, donde es considerada como "la primera de las libertades".

\section{Referencias}

Casas Becerra, L. \& Lawson, D. (2016). Debates y reflexiones en torno a la despenalización del aborto en Chile. Centro de Derechos Humanos UDP.

Celis, A. M. \& Zárate, S. (2015, diciembre). Libertad de enseñanza y libertad religiosa: los establecimientos escolares con orientación religiosa en Chile. Temas de la Agenda Pública, 10(84).

Chapman, N. S. (2013). Disentangling conscience and religión. University of Illinois Law Review, (4), 1457-1502.

Ciáurriz, M. J. (1994). El contenido del derecho fundamental de libertad religiosa. In V.V.A.A. Tratado de Derecho Eclesiástico (pp. 429-468). Pamplona: Eunsa.

Constitución Política de La República de Chile de 1980. Disponible en: https://www.leychile.cl/ Navegar?idNorma $=242302$

Declaración Universal de Derechos Humanos. Adoptada por la Asamblea General de las Naciones Unidas el 10 de diciembre de 1948. Disponible en http://www.un.org/es/universal-declaration-human-rights/

Del Picó Rubio, J. (2017, abril). Evaluación del sistema jurídico de reconocimiento del fenómeno religioso en Chile 1999-2013. Revista Latinoamericana de Derecho y Religión, 3(1). Disponible en: http://www.revistalatderechoyreligion.com/ojs/ojs-2.4.6/index.php/RLDR/article/view/52 
Escobar Delgado, R. A. (2017). The right to freedom of religion and cults in Colombia: evolution in constitutional jurisprudence 1991-2015. Prolegómenos, 20(39), 125-138.

Fediakova, E. \& Parker, C. (2009). Evangélicos en Chile Democrático (1990-2008): Radiografía al centésimo aniversario. Revista Cultura y Religión, III(2).

Flere, S., Lavric, M., \& Djordjevic, D. B. (2017, septiembre). Religious References in the Constitutions of European Post-Communist Countries and Ethno-symbolism. Journal of Church and State, 59(3), 466-488.

Gas Aixendri, M. (2012). Apostasía y libertad religiosa. Conceptualización jurídica del abandono confesional. Granada: Comares.

González Merlano, G. (2015, julio). Perspectiva jurídica de la libertad religiosa y la libertad de conciencia. Revista de Derecho de la Universidad Católica del Uruguay, 10(11), 81-104.

Instituto Nacional de Derechos Humanos de Chile (2016). Informe anual sobre la situación de los derechos humanos. Disponible en: http://indh.cl/wp-content/uploads/2016/12/Informe-Anual-INDH-2016.pdf

Koppelmann, A. (2006). Is it fair to give religion special treatment? University of Illinois Law Review, (3), 571-604

Martín de Agar, J. T. (2003). Los principios del Derecho Eclesiástico del Estado. Revista de Derecho de la Pontificia Universidad Católica de Valparaíso, XXIV, 333-344.

Martín de Agar, J. T. (2016, abril). Opción pacticia y libertad religiosa institucional. Anuario de Derecho Canónico, (5), 189-206.

Olmos Ortega, M. E. (2016). El derecho a la libertad religiosa: un tema de nuestro tiempo. Lección magistral en la apertura del año judicial del Tribunal Eclesiástico Metropolitano de Valencia. Disponible en: http://www.archivalencia.org/contenido.php? $\mathrm{a}=6 \& \mathrm{pad}=6 \& \operatorname{modulo}=37 \& \mathrm{i}-$ $\mathrm{d}=13309$ \&pagina $=1$

Pankhurst, J. G. \& Kilp, A. (2013). Religion, the Russian Nation and the State: Domestic and International Dimension: an Introduction. Religion, State and Society, 41(3), 226-243.

Papa Francisco. Discurso en el encuentro por la libertad religiosa con la comunidad hispana y otros inmigrantes, pronunciado en Filadelfia el 26 de septiembre de 2015. Disponible en: http://w2.vatican.

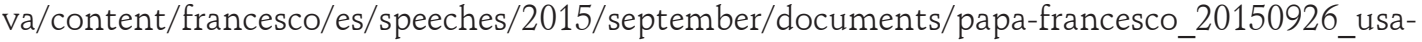
-liberta-religiosa.html

Parker, C. ¿̇América Latina ya no es católica? El incremento del pluralismo cultural y religioso. En Llambias-Wolff, J. (Ed.), Latin America: questions and perspectives (pp. 205-232). York: York University Bookstore, 2013.

Precht, J. (2015, octubre). Libertad religiosa y ley de cultos. Primera parte. Diario Constitucional. Disponible en: http://www.diarioconstitucional.cl/articulos/libertad-religiosa-y-ley-de-cultos-primera-parte

Precht, J. (2015). Libertad religiosa y ley de cultos. Segunda parte. Boletín Jurídico, XI(3), 40-43. 
Rossell, J. (2015). La promoción de la libertad religiosa a través de la cooperación entre el Estado y las confesiones religiosas. In Martínez de Codes, R. M. \& Fajardo Arturo, L. A. Tendencias secularizadoras en un mundo globalizado (pp. 99-127). Bogotá: Universidad Sergio Arboleda. Disponible en: http://repository.usergioarboleda.edu.co/bitstream/handle/11232/751/Tendencias\%20 secularizadoras.pdf? sequence $=1$ \& is Allowed $=y$

Rubio López, J. I. (2006). La primera de las libertades. La libertad religiosa en los EE.UU. durante la Corte Rehnquist (1986-2005): una libertad en tensión. Pamplona: Eunsa.

Salinas, C. (2006). El influjo del Derecho canónico en el Código Civil de la República de Chile. Valparaíso: Ediciones Universitarias de Valparaíso.

Salinas, C. (2016). El Derecho Canónico en Chile. Siglo XIX. Valparaíso: Ediciones Universitarias de Valparaíso.

Sánchez-Lasheras, M. (2016, abril). Derecho y factor religioso en Chile y en el Perú: ว̀hacia la gestión pública de la diversidad religiosa? Revista Chilena de Derecho, 43(1), 173-174.

United States Comission on International Religious Freedom (2017). Annual Report. Disponible en: http://www.uscirf.gov/reports-briefs/annual-report

U.S. Department of State (2015). Bureau of Democracy, Human Rights, and Labor. International Religious Freedom Report. Disponible en: https://2009-2017.state.gov/j/drl/rls/irf/2015/ wha/256341.htm

Data da submissão: 06 de dezembro de 2016 Avaliado em: 09 de abril de 2018 (AVALIADOR A) Avaliado em: 10 de abril de 2018 (AVALIADOR B) Avaliado em: 10 de abril de 2018 (AVALIADOR C) Aceito em: 15 de abril de 2018 
